\title{
不同工况下活塞二阶运动和活塞裙部润滑研究
}

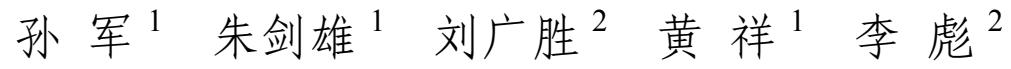 \\ 朱少禹 ${ }^{1}$ 苗恩铭 $^{3}$ 郝飞飞 $^{1}$ 胡新亮 ${ }^{2}$ 李 $^{1}$ 悦 $^{1}$ \\ (1. 合肥工业大学汽车与交通工程学院 合肥 230009; \\ 2. 合肥工业大学机械工程学院 合肥 230009 ; \\ 3. 合肥工业大学仪器科学与光电工程学院 合肥 230009)
}

\begin{abstract}
摘要: 目前研究内燃机活塞二阶运动和活塞裙-缸套摩擦副润滑时一般仅针对标定工况进行, 不考虑实际使用内燃机工作中工 况的不断变化。另外, 分析中一般都假设活塞裙-缸套摩擦副在一个内燃机工作循环中处于充分润滑状况。以某四行程内燃机 为研究对象, 基于实测的气缸压力, 考虑实际润滑油流动确定摩擦副的润滑状况, 进行不同内燃机工况下的活塞二阶运动和 活塞裙-缸套摩擦副润滑分析。结果表明, 不同工况下的活塞二阶运动性能和活塞裙-缸套摩擦副润滑性能都存在差异, 活塞 二阶运动性能和活塞裙-缸套摩擦副润滑性能的最不利情况不一定出现在标定工况。因此, 内燃机活塞组件设计时, 如果仅分 析标定工况下的活塞二阶运动和活塞裙-缸套摩擦副润滑性能将不够全面合理, 需要进行不同工况下活塞二阶运动和活塞裙-缸 套摩擦副润滑性能的分析计算。
\end{abstract}

关键词: 内燃机; 活塞; 二阶运动; 活塞裙; 润滑; 工况

中图分类号: TK40

\section{On the Piston Secondary Motion of Piston and Lubrication of Piston Skirt at Different Operating Conditions}

\author{
SUN Jun $^{1} \quad$ ZHU Jianxiong $^{1} \quad$ LIU Guangsheng $^{2} \quad$ HUANG Xiang $^{1} \quad$ LI Biao $^{2} \quad$ ZHU Shaoyu $^{1}$ \\ MIAO Enming ${ }^{3} \quad$ HAO Feifei ${ }^{1} \quad$ HU Xinliang $^{2} \quad$ LI Yue $^{1}$
}

(1. School of Automotive and Transportation Engineering, Hefei University of Technology, Hefei 230009;

2. School of Mechanical Engineering, Hefei University of Technology, Hefei 230009;

3. School of Instrument Science and Opto-electronics Engineering,

Hefei University of Technology, Hefei 230009)

\begin{abstract}
Current research on the piston secondary motion and the lubrication of piston skirt-cylinder liner friction conjunction for internal combustion engine is generally carried out in rated operating condition, and the constant change of operating condition for actual used internal combustion engine is not considered. In addition, the piston skirt-cylinder liner friction conjunction is usually supposed to be lubricated sufficiently in all strokes of an operating cycle of internal combustion engine in current analysis. A four-stroke internal combustion engine is taken as the studying object, based on measured cylinder pressure, the piston secondary motion and the lubrication of piston skirt-cylinder liner friction conjunction considering the lubrication status determined by the actual flow of lubricating oil in different operating conditions of internal combustion engine are analyzed. The results show that there are the differences of the piston secondary motion and the lubricating characteristics of piston skirt-cylinder liner friction conjunction in different operating conditions, and the worst condition may not be in rated operating condition. Therefore, it is not comprehensive and reasonable that the piston secondary motion and the lubrication of piston skirt-cylinder liner friction conjunction are analyzed only in rated operating condition, and it is necessary to calculate the piston secondary motion and the lubrication of piston skirt-cylinder liner friction conjunction in different operating conditions in the design of piston set.
\end{abstract}

* 国家自然科学基金(50975073，51490660，51490661)和内燃机可靠性国 家重点实验室开放基金(skler-201708)资助项目。20180913 收到初稿, 20190112 收到修改稿 
Key words: IC engine; piston; secondary motion; piston skirt; lubrication; operating condition

\section{0 前言}

作为主要零件, 活塞对往复式内燃机的可靠工 作有重要影响。内燃机工作时, 活塞在气缸中进行 上下往复主运动的同时, 还存在绕活塞销摆动的二 阶运动。活塞二阶运动会使活塞拍击气缸, 产生噪 声, 影响活塞与气缸套间的润滑、摩擦和磨损。活 塞裙部的主要功能是引导活塞运动, 活塞裙部-缸套 摩擦副的润滑直接影响其工作状况。

随着相关技术的进步和研究的不断深入，近年 来内燃机活塞二阶运动和活塞裙-缸套摩擦副润滑 分析中考虑的方面日益完善, 预测更加精确。 DURSUNKAYA 等 ${ }^{[1-4]}$ 研究了活塞裙部和缸套变形 对活塞二阶运动的影响, MANSOURI 等 ${ }^{[5-7]}$ 针对活 塞裙部型线等活塞设计参数的影响进行了研究, LIU 等 ${ }^{[8-9]}$ 探讨了活塞销偏置的影响, ZHANG 等 ${ }^{[10-11]}$ 分析了连杆 - 曲轴-飞轮系统变惯量的影响, MURAKAMI 等 ${ }^{[2]}$ 考虑活塞、气缸和缸盖的变形耦 合进行了分析, MENG 等 ${ }^{[13-14]}$ 研究了连杆、曲轴、 飞轮和活塞动力学的耦合影响。KNOLL 等 ${ }^{[15]}$ 研究 了活塞裙部润滑分析中的载荷和形状处理, LI 等 ${ }^{[16]}$ 分析了活塞销位置、间隙和润滑油黏度对裙部润滑 的影响, ZHU 等 ${ }^{[17]}$ 提出了裙部混合润滑分析的基 本模型，杨俊伟等 ${ }^{[3-4,18-20]}$ 等分别探讨了裙部和缸 套弹性和热变形的影响, GUNELSU 等 ${ }^{[6-7,21]}$ 分析了 裙部型面的影响, 余志壮等 ${ }^{[22-23]}$ 分别研究了气缸套 失圆和油膜惯性的影响, QASIM 等 ${ }^{[24-25]}$ 研究了起动 工况小径向间隙下非牛顿润滑油的活塞裙弹流润 滑, MAHDAVI 等 ${ }^{[26]}$ 研究了磁流变液体对裙部润滑 的影响, NING 等 ${ }^{[27]}$ 分析了多级润滑油剪切稀化的 影响, MENG 等 ${ }^{[28]}$ 研究了连杆设计参数对裙部润滑 的影响。

然而, 已开展的内燃机活塞二阶运动和活塞裙缸套摩擦副润滑性能研究一般仅针对标定工况进 行, 不考虑实际使用内燃机工作中工况的不断变化。 另外, 分析中一般都假设活塞裙-缸套摩擦副在一个 内燃机工作循环中处于充分润滑情况。因此, 有必 要对不同工况下内燃机活塞二阶运动和活塞裙-缸 套摩擦副润滑性能进行研究, 这将有助于完善内燃 机活塞二阶运动和活塞裙-缸套摩擦副润滑分析理 论, 为设计提供更全面的参考依据。

本文针对某四行程内燃机, 计及润滑油流动影 响, 分析不同内燃机工况下的活塞二阶运动和活塞
裙-缸套摩擦副润滑性能, 研究内燃机工况对活塞二 阶运动和活塞裙-缸套摩擦副润滑性能的影响。

\section{1 方程和公式}

\section{1 活塞二阶运动方程}

活塞的二阶运动受活塞惯性力、缸内压力、 摩擦副油膜压力和摩擦力等的综合作用影响。图 1 为活塞结构及其受力示意图, 其中 $F_{\mathrm{g}}$ 为气缸压力 的作用力, $F_{\mathrm{py}}$ 和 $F_{\mathrm{cy}}$ 为活塞销和活塞的惯性力, $F$ 为润滑油膜反力和微凸体接触力的合力, $F_{\mathrm{f}}$ 为润 滑油膜和微凸体接触摩擦力的合力, $F_{\text {ip }}$ 和 $F_{\text {ic }}$ 为 活塞二阶运动产生的活塞销和活塞惯性力, $F_{\text {con }}$ 为连杆力, $M$ 为润滑油膜反力矩和微凸体接触力 矩的合力矩, $M_{\mathrm{f}}$ 为润滑油膜和微凸体接触摩擦力 矩的合力矩, $M_{\mathrm{ic}}$ 为活塞二阶运动产生的活塞惯性 力矩。 $L$ 为活塞裙部长度, $\Phi$ 为连杆摆角。
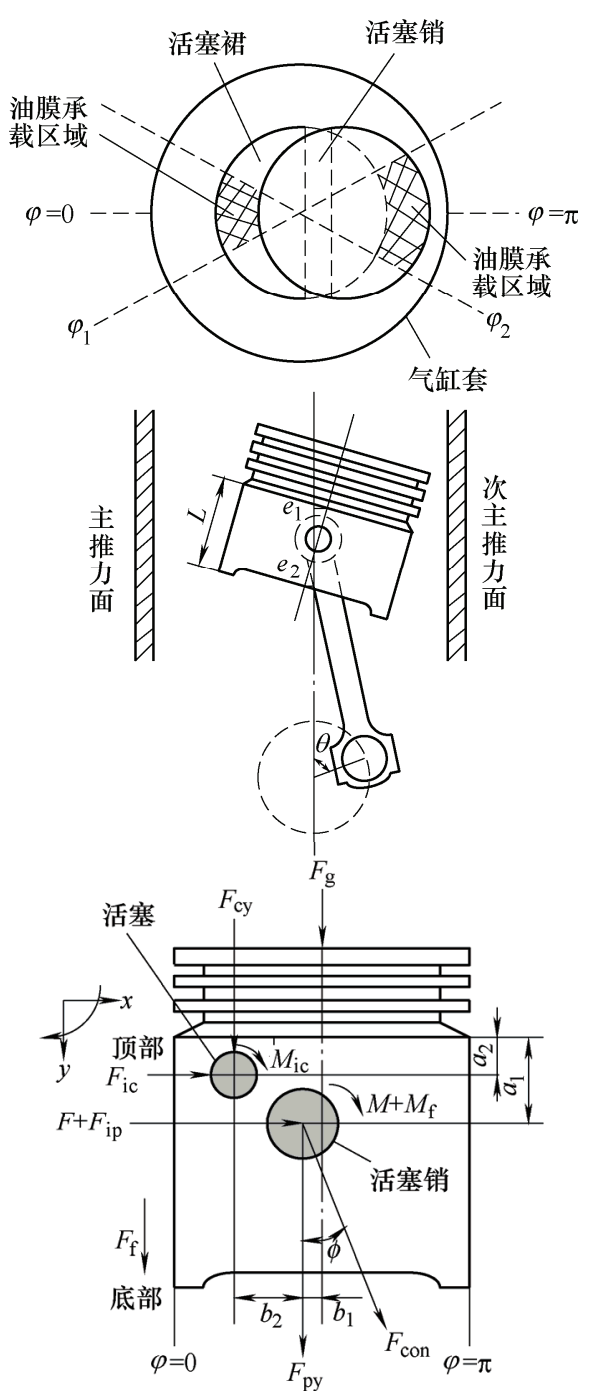

图 1 活塞的位置和受力 
根据活塞的受力和运动情况分析, 得到活塞二 阶运动方程为

$$
\begin{gathered}
\left(\begin{array}{cc}
m_{1}\left(1-\frac{a_{1}}{L}\right)+m_{2}\left(1-\frac{a_{2}}{L}\right) \quad m_{1} \frac{a_{1}}{L}+m_{2} \frac{a_{2}}{L} \\
\frac{I_{1}}{L}+m_{2}\left(a_{1}-a_{2}\right)\left(1-\frac{a_{2}}{L}\right) & m_{2}\left(a_{1}-a_{2}\right) \frac{a_{2}}{L}-\frac{I_{1}}{L}
\end{array}\right) \\
\left(\begin{array}{c}
e_{\mathrm{t}}^{\prime \prime} \\
e_{\mathrm{b}}^{\prime \prime}
\end{array}\right)=\left(\begin{array}{c}
F+F_{\mathrm{s}}+F_{\mathrm{f}} \tan \phi \\
M+M_{\mathrm{s}}+M_{\mathrm{f}}
\end{array}\right)
\end{gathered}
$$

式中, $m_{1}$ 为活塞销质量, $a_{1}$ 为活塞销孔中心与活塞 裙顶部之间的距离, $m_{2}$ 为活塞质量, $a_{2}$ 为活塞质心 与活塞裙顶部之间的距离, $I_{1}$ 为活塞转动惯量, $e_{\mathrm{t}}$ 为活塞裙顶部中心的位移, $e_{\mathrm{b}}$ 为活塞裙底部中心的 位移。

$$
\begin{gathered}
F=F_{\mathrm{o}}+F_{\mathrm{c}} \\
F_{\mathrm{s}}=\left(F_{\mathrm{g}}+F_{\mathrm{py}}+F_{\mathrm{cy}}\right) \tan \phi
\end{gathered}
$$

式中, $F_{\mathrm{g}}$ 为气缸压力的作用力, $F_{\mathrm{py}}$ 为活塞销惯性力, $F_{\text {cy }}$ 为活塞惯性力。

$$
F_{\mathrm{f}}=F_{\mathrm{fo}}+F_{\mathrm{fc}}
$$

式中, $F_{\mathrm{fo}}$ 为润滑油摩擦力, $F_{\mathrm{fc}}$ 为粗糙表面接触摩 擦力。

$$
M=M_{\mathrm{o}}-M_{\mathrm{c}}
$$

式中, $M_{\mathrm{o}}$ 为油膜压力产生的承载力矩, $M_{\mathrm{c}}$ 为粗粘 表面接触力矩。

$$
M_{\mathrm{s}}=F_{\mathrm{g}} b_{1}-F_{\mathrm{cy}} b_{2}
$$

式中, $b_{1}$ 为活塞销孔偏心距, $b_{2}$ 为活塞销孔中心与 活塞质心之间的距离。

$$
M_{\mathrm{f}}=M_{\mathrm{fo}}+M_{\mathrm{fc}}
$$

式中, $M_{\mathrm{fo}}$ 为润滑油摩擦力矩, $M_{\mathrm{fc}}$ 为粗䊁表面接触 摩擦力矩。

\subsection{Reynolds 方程}

采用平均 Reynolds 方程 ${ }^{[29]}$ 求解活塞裙-缸套摩 擦副间的流体润滑。

$$
\begin{gathered}
\frac{\partial}{\partial x}\left(\Phi_{\mathrm{x}} \frac{h^{3}}{12 \mu} \frac{\partial p}{\partial x}\right)+\frac{\partial}{\partial y}\left(\Phi_{\mathrm{y}} \frac{h^{3}}{12 \mu} \frac{\partial p}{\partial y}\right)= \\
\frac{u}{2} \frac{\partial \bar{h}}{\partial y}+\frac{u}{2} \sigma \frac{\partial \Phi_{\mathrm{s}}}{\partial y}+\frac{\partial \bar{h}}{\partial t}
\end{gathered}
$$

式中, $p$ 为油膜压力; $\Phi_{\mathrm{x}} 、 \Phi_{\mathrm{y}} 、 \Phi_{\mathrm{s}}$ 为压力和剪切流量 因子 ${ }^{[30] ;} h$ 为不计表面粗䊁的油膜厚度; $\bar{h}$ 为油膜厚 度平均值; $u$ 为活塞沿气缸中心的运动速度; $\mu$ 为润 滑油黏度; $\sigma$ 为综合表面粗鋉度, $\sigma=\sqrt{\sigma_{\mathrm{s}}^{2}+\sigma_{\mathrm{c}}{ }^{2}}, \sigma_{\mathrm{s}}$ 、 $\sigma_{\mathrm{c}}$ 分别为活塞裙和缸套表面粗粘度。

\section{3 油膜厚度}

$$
\begin{gathered}
h=c+e_{\mathrm{t}} \cos \varphi+\left[e_{\mathrm{b}}-e_{\mathrm{t}}\right] \frac{y}{L} \cos \varphi+ \\
f(\varphi, y)+\delta_{\mathrm{s}}(\varphi, y)+\delta_{\mathrm{c}}(\varphi, y)
\end{gathered}
$$

式中, $c$ 为活塞裙部与缸套之间的间隙; $f(\varphi, y)$ 为活 塞裙部表面曲率沿轴向的变化函数; $\delta_{\mathrm{s}}(\varphi, y)$ 和 $\delta_{\mathrm{c}}(\varphi$, $y$ )分别为活塞裙部和缸套表面的弹性变形。

\section{4 油膜承载力和力矩}

$$
\begin{gathered}
F_{\mathrm{o}}=\iint p \cos \varphi \mathrm{d} x \mathrm{~d} y \\
M_{\mathrm{o}}=\iint p(a-y) \cos \varphi \mathrm{d} x \mathrm{~d} y
\end{gathered}
$$

\section{5 润滑油摩擦力和力矩}

$$
\begin{gathered}
F_{\mathrm{fo}}=\iint \tau \mathrm{d} x \mathrm{~d} y \\
M_{\mathrm{fo}}=\iint \tau\left(R \cos \varphi-C_{\mathrm{p}}\right) \mathrm{d} x \mathrm{~d} y
\end{gathered}
$$

式中, $R$ 为活塞半径。

$$
\tau=-\frac{\mu u}{\bar{h}}\left(\Phi_{\mathrm{f}}+\Phi_{\mathrm{fs}}\right)+\Phi_{\mathrm{fp}} \frac{\bar{h}}{2} \frac{\partial p}{\partial y}
$$

式中, $\Phi_{\mathrm{f}} 、 \Phi_{\mathrm{fs}} 、 \Phi_{\mathrm{fp}}$ 为平均切应力因子 ${ }^{[30]}$ 。

\section{6 粗粘表面的接触力和力矩}

采用 GREENWOOD 等 ${ }^{[31}$ 提出的微凸体接触模 型研究活塞裙部与缸套间的接触问题。

$$
\begin{gathered}
F_{\mathrm{c}}=\iint_{A_{\mathrm{c}}} p_{\mathrm{c}}(x, y) \cos \varphi \mathrm{d} x \mathrm{~d} y \\
M_{\mathrm{c}}=\iint_{A_{\mathrm{c}}} p_{\mathrm{c}}(x, y)(a-y) \cos \varphi \mathrm{d} x \mathrm{~d} y
\end{gathered}
$$

式中, $p_{\mathrm{c}}$ 为粗粘表面接触压力平均值, $A_{\mathrm{c}}$ 为实际接 触面积。

$$
\begin{gathered}
p_{\mathrm{c}}=\left(\frac{16 \sqrt{2}}{15}\right) \pi(\eta \beta \delta)^{2} E^{\prime} \sqrt{\frac{\delta}{\eta}} F_{5 / 2}(H) \\
A_{\mathrm{c}}=\pi^{2}(\eta \beta \sigma)^{2} A F_{2}(H)
\end{gathered}
$$

式中, $\eta$ 为粗粘表面微凸体密度, $\beta$ 为微凸体曲率半 径, $A$ 为不计表面粗糙的接触面积, $E^{\prime}$ 为综合弹性 模量。 $\eta \beta \sigma$ 和 $\sigma / \eta$ 分别取值 0.04 和 0.001 。

$$
\begin{gathered}
H=\frac{h}{\sigma} \\
F_{\mathrm{n}}(H)=\frac{1}{\sqrt{2 \pi}} \int_{H}^{\infty}(s-H)^{n} \exp \left(\frac{-s^{2}}{2}\right) \mathrm{d} s
\end{gathered}
$$

\section{7 粗糙表面的接触摩擦力和力矩}

$$
\begin{gathered}
F_{\mathrm{fc}}=-\frac{|u|}{u} \iint_{A_{\mathrm{c}}} \tau_{\mathrm{a}}(x, y) \mathrm{d} x \mathrm{~d} y \\
M_{\mathrm{fc}}=\frac{|u|}{u} \iint_{A_{\mathrm{c}}} \tau_{\mathrm{a}}(x, y)\left(R \cos \varphi-b_{1}\right) \mathrm{d} x \mathrm{~d} y
\end{gathered}
$$




$$
\tau_{\mathrm{a}}=\tau_{0}+\mu_{\mathrm{f}} \cdot p_{\mathrm{c}}
$$

式中, $\tau_{0}$ 为综合切应力因数 (取 $2 \mathrm{MPa}$ ), $\mu_{\mathrm{f}}$ 为边界摩 擦因数(取 0.08 )。

\section{8 润滑油流动方程}

在内燃机工作循环中, 活塞由上止点向下止点 移动时, 由于前一行程活塞向上止点移动, 大量润 滑油被飞溅到气缸壁表面, 活塞裙部的润滑油充足, 处于充分润滑的状况。而活塞由下止点向上止点移 动时, 活塞裙部润滑只有活塞向下止点移动过后附 着在气缸壁面的润滑油。

活塞向下止点移动过后附着在气缸壁面的润滑 油量 $V_{\mathrm{m}}$ 为

$$
V_{\mathrm{m}}=V_{1}+V_{2}+V_{3}
$$

式中, $V_{1}$ 为活塞裙部与缸套间的润滑油量, $V_{2}$ 为活 塞下行带走的润滑油量, $V_{3}$ 为压力差产生的润滑油 量 ${ }^{[32]}$ 。

$$
\begin{gathered}
V_{1}=\iint_{A_{\mathrm{m}}} \Delta x \Delta y h \mathrm{~d} x \mathrm{~d} y \\
V_{2}=\iint_{A_{\mathrm{m}}}-\Delta x \frac{u}{2} h \Delta t \mathrm{~d} x \mathrm{~d} y \\
V_{3}=\iint_{A_{\mathrm{m}}} \Delta x \frac{h^{3}}{12 \mu \Delta y} \Delta p \Delta t \mathrm{~d} x \mathrm{~d} y
\end{gathered}
$$

式中, $A_{\mathrm{m}}$ 为附着润滑油的面积, $\Delta x 、 \Delta y$ 为周向和 轴向网格的边长, $\Delta t$ 为时间步长。

\section{2 分析方法}

(1) 输入计算参数。包括活塞等结构和表面参 数、内燃机转速、气缸压力和润滑油动力黏度等。

(2) 取内燃机工作循环的进气上止点为计算起 始点, 曲轴转角 $\theta=0^{\circ} \mathrm{CA}$, 计算步长 $\Delta \theta=1^{\circ} \mathrm{CA}$, 同时设定此时刻的初值 $e_{\mathrm{t}}\left(0^{\circ} \mathrm{CA}\right)=e_{\mathrm{b}}\left(0^{\circ} \mathrm{CA}\right)=0$, $e_{\mathrm{t}}^{\prime}\left(0^{\circ} \mathrm{CA}\right)=e_{\mathrm{b}}^{\prime}\left(0^{\circ} \mathrm{CA}\right)=0$ 。

(3) 采用有限差分法和超松弛迭代法相结合的 方法求解 Reynolds 方程, 计算油膜压力。

（4）应用式(10) (13)求解活塞裙部油膜反力、 油膜反力矩、摩擦力和摩擦力矩。

(5) 应用式(15)、(16)、(20)和(21)求解活塞裙部 粗粮表面的接触力、接触力矩、接触摩擦力和摩擦 力矩。

（6）结合缸内气体压力产生的作用力, 应用 Broyden 方法 ${ }^{[33]}$ 求解活塞二阶运动方程。

(7) 如果 $\theta>720^{\circ} \mathrm{CA}$, 且满足收玫条件, 即

$$
\begin{aligned}
& \left|\frac{e_{\mathrm{t}}\left(720^{\circ}\right)-e_{\mathrm{t}}\left(0^{\circ}\right)}{e_{\mathrm{t}}\left(0^{\circ}\right)}\right| \leqslant 0.001 \\
& \left|\frac{e_{\mathrm{b}}\left(720^{\circ}\right)-e_{\mathrm{b}}\left(0^{\circ}\right)}{e_{\mathrm{b}}\left(0^{\circ}\right)}\right| \leqslant 0.001 \\
& \left|\frac{e_{\mathrm{t}}^{\prime}\left(720^{\circ}\right)-e_{\mathrm{t}}^{\prime}\left(0^{\circ}\right)}{e_{\mathrm{t}}^{\prime}\left(0^{\circ}\right)}\right| \leqslant 0.001 \\
& \left|\frac{e_{\mathrm{b}}^{\prime}\left(720^{\circ}\right)-e_{\mathrm{b}}^{\prime}\left(0^{\circ}\right)}{e_{\mathrm{b}}^{\prime}\left(0^{\circ}\right)}\right| \leqslant 0.001
\end{aligned}
$$

则循环计算结束, 输出结果。否则, 令

$$
e_{\mathrm{t}}\left(0^{\circ} \mathrm{CA}\right)=e_{\mathrm{t}}\left(720^{\circ} \mathrm{CA}\right), e_{\mathrm{b}}\left(0^{\circ} \mathrm{CA}\right)=e_{\mathrm{b}}\left(720^{\circ} \mathrm{CA}\right)
$$

$e_{\mathrm{t}}^{\prime}\left(0^{\circ} \mathrm{CA}\right)=e_{\mathrm{t}}^{\prime}\left(720^{\circ} \mathrm{CA}\right), e_{\mathrm{b}}^{\prime}\left(0^{\circ} \mathrm{CA}\right)=e_{\mathrm{b}}^{\prime}\left(720^{\circ} \mathrm{CA}\right)$ 转到步骤(2), 进行下一个循环的活塞二阶运动和活 塞裙部润滑计算。

图 2 为分析计算的流程图。

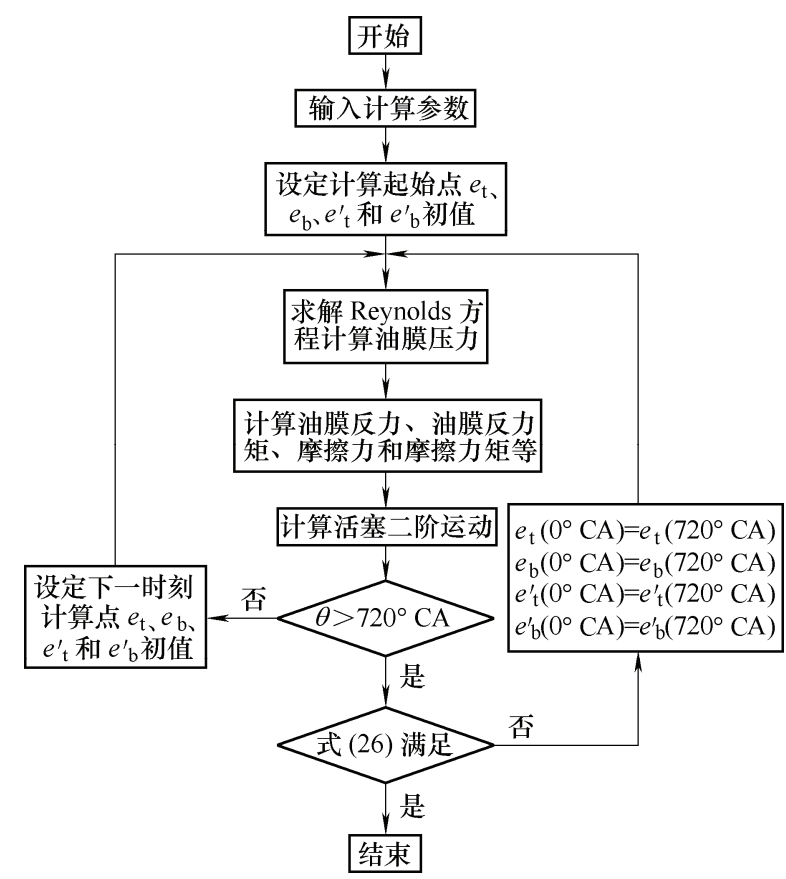

图 2 计算流程图

\section{3 结果和分析}

以某四行程内燃机为对象, 分析不同工况(转速 和负荷)下的活塞二阶运动和活塞裙-缸套摩擦副润 滑性能。表 1 为该内燃机的主要参数。选取九种内 燃机工况, 分别为 $1200 \mathrm{r} / \mathrm{min}$ (低转速)、2200 $\mathrm{r} / \mathrm{min}$ (中等转速, 最大转矩点转速)和 $3200 \mathrm{r} / \mathrm{min}$ (高 转速, 标定转速)下, 负荷 $0 \%$ (小负荷)、负荷 $60 \%$ (中 等负荷)、负荷 $100 \%$ (全负荷)。在内燃机台架上试 验获取各种工况的气缸压力。 
表 1 主要参数

\begin{tabular}{|c|c|}
\hline 参数 & 数值 \\
\hline 标定功率 $P_{\mathrm{e}} / \mathrm{kW}$ & 76 \\
\hline 标定转速 $n /(\mathrm{r} / \mathrm{min})$ & 3200 \\
\hline 活塞销质量 $m_{1} / \mathrm{kg}$ & 0.57 \\
\hline 活塞质量 $m_{2} / \mathrm{kg}$ & 1.13 \\
\hline 活塞转动惯量 $I_{1} /\left(\mathrm{N} \cdot \mathrm{m}^{2}\right)$ & 0.009 \\
\hline 活塞半径 $R \mathrm{~mm}$ & 52.4 \\
\hline 活塞裙部长度 $L / \mathrm{mm}$ & 66.3 \\
\hline 活塞销孔中心与活塞裙顶部之间的距离 $a_{1} / \mathrm{mm}$ & 20.75 \\
\hline 活塞质心与活塞裙顶部之间距离 $a_{2} / \mathrm{mm}$ & 2 \\
\hline 活塞裙表面粗粘度 $\sigma_{\mathrm{s}} / \mu \mathrm{m}$ & 2.5 \\
\hline 缸套表面粗糙度 $\sigma_{\mathrm{c}} / \mu \mathrm{m}$ & 0.8 \\
\hline 润滑油黍度 $\mu /(\mathrm{Pa} \cdot \mathrm{s})$ & 0.01 \\
\hline
\end{tabular}

图 3 9 分别为负荷 $0 \% 、 60 \%$ 和 $100 \%$, 不同转 速(1 $200 \mathrm{r} / \mathrm{min} 、 2200 \mathrm{r} / \mathrm{min}$ 和 $3200 \mathrm{r} / \mathrm{min}$ )下, 内燃 机一个工作循环中活塞二阶运动和活塞裙-缸套摩 擦副润滑性能参数的变化情况。表 $2 \sim 4$ 分别为一个 工作循环中, 活塞裙-缸套摩擦副最小油膜厚度的最 小值、最大油膜压力的最大值和平均摩擦功率。

\section{1 相同负荷不同转速时}

由图 3 和图 4 可见, 内燃机负荷较小和中等(负 荷 $0 \%$ 和 $60 \%$ ) 时, 不同转速下, 活塞二阶运动横向 位移的最大值都出现在燃烧上止点附近, 此时活塞 的摆动和倾斜角最大; 内燃机负荷较高 $(100 \%$ 负荷) 时, 在中等和较高转速 (2 $200 \mathrm{r} / \mathrm{min}$ 和 $3200 \mathrm{r} / \mathrm{min}$ ) 下, 活塞横向位移的最大值也出现在燃烧上止点附 近。但是, 内燃机负荷较高(负荷 $100 \%$ ) 时, 在较低 转速(1 $200 \mathrm{r} / \mathrm{min}$ )下, 活塞横向位移的最大值位于进 气上止点附近, 此处的活塞摆动和倾斜角最大。

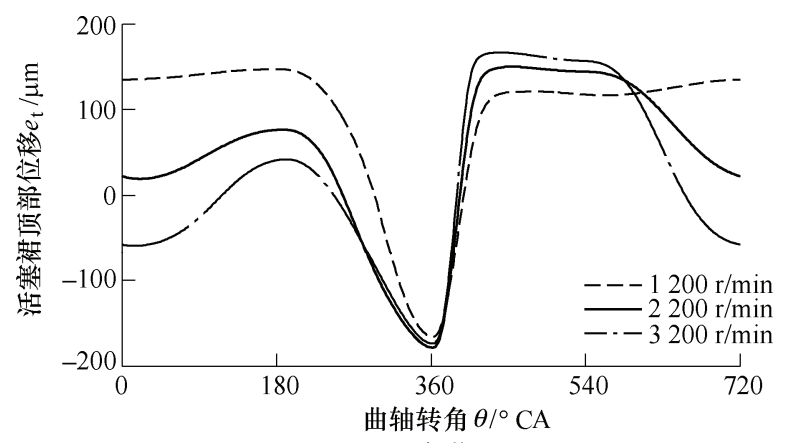

(a) 负荷 $0 \%$

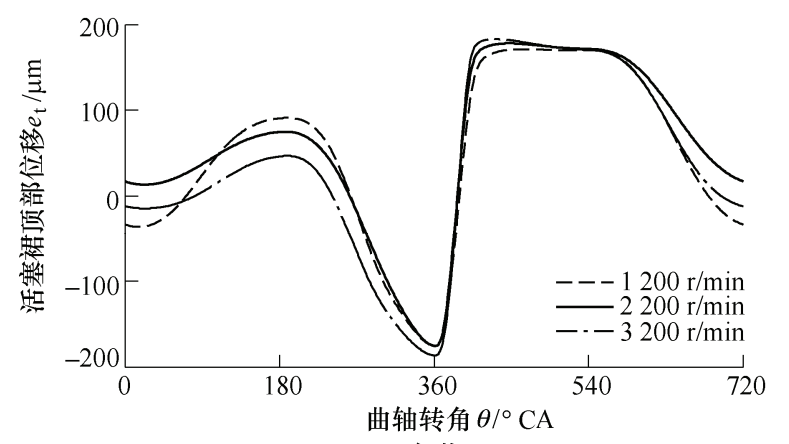

(b) 负荷 $60 \%$

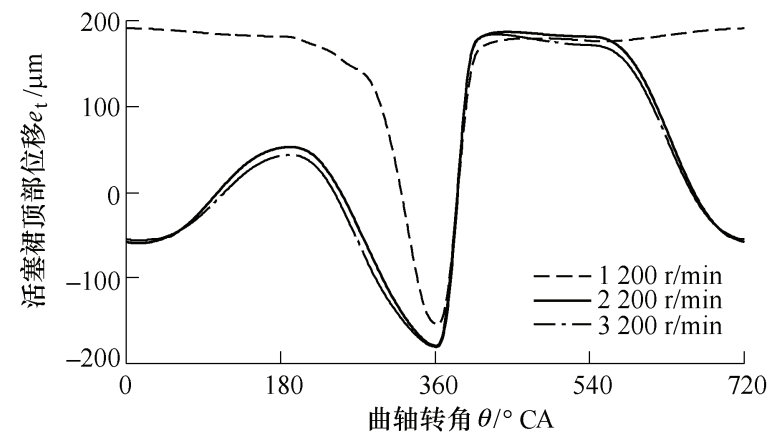

(c) 负荷 $100 \%$

图 3 活塞裙顶部位移

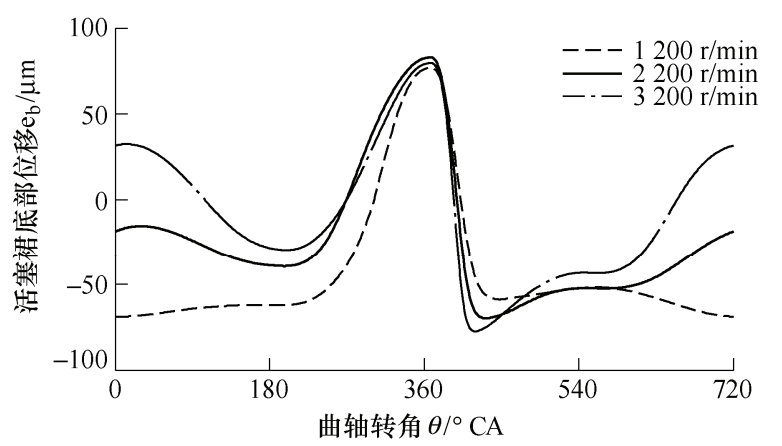

(a) 负荷 $0 \%$

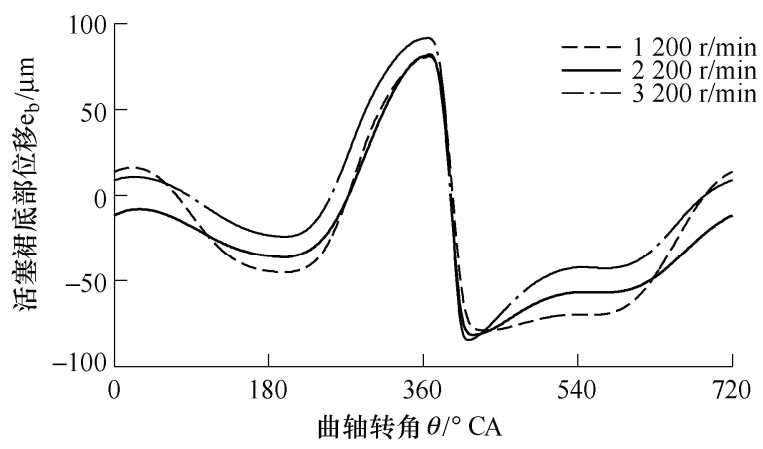

(b) 负荷 $60 \%$

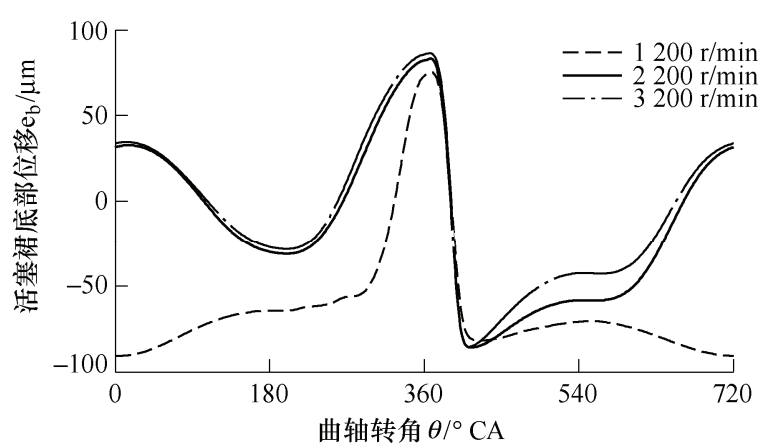

(c) 负荷 $100 \%$

图 4 活塞裙底部位移

内燃机中等负荷(负荷 $60 \%$ ) 时, 随转速变化, 工作循环中不同时刻活塞横向位移的方向和大小基 本一致，活塞的摆动和倾斜方位基本相同; 内燃机 较高负荷(负荷 100\%)时，在中等和较高转速(2 200 $\mathrm{r} / \mathrm{min}$ 和 $3200 \mathrm{r} / \mathrm{min}$ )下, 工作循环中不同时刻活塞 横向位移的方向和大小也基本一致。但是, 内燃 机负荷较小 (负荷 $0 \%$ )时, 在不同转速下, 进气行 
程和排气行程中活塞横向位移的方向和大小存 在不同, 活塞的摆动和倾斜方位出现不一致; 内 燃机较高负荷(负荷 $100 \%$ ) 时, 在较低转速(1 200 $\mathrm{r} / \mathrm{min}$ )下, 进气行程和排气行程中活塞横向位移的方 向和大小与中高转速 $(2200 \mathrm{r} / \mathrm{min}$ 和 $3200 \mathrm{r} / \mathrm{min})$ 下 相差较大，活塞的摆动和倾斜方位将明显不一致。

如图 5 和图 6 所示, 内燃机不同负荷时，在不 同转速下, 活塞横向移动速度在一个内燃机工作循 环中的变化趋势基本相同, 活塞横向移动速度的最 大值一般都出现在膨胀行程靠近燃烧上止点附近, 此时活塞的摆动和冲击剧烈; 随着转速提高, 活塞 二阶运动横向移动速度的最大值将增加, 活塞摆动 和冲击情况将加剧。另外, 内燃机负荷较小和较高 (负荷 $0 \%$ 和 100\%)时, 在较低转速(1 $200 \mathrm{r} / \mathrm{min}$ )下, 压缩行程靠近上止点区域的活塞横向移动速度也相 对较大, 将会增加活塞的摆动幅度。

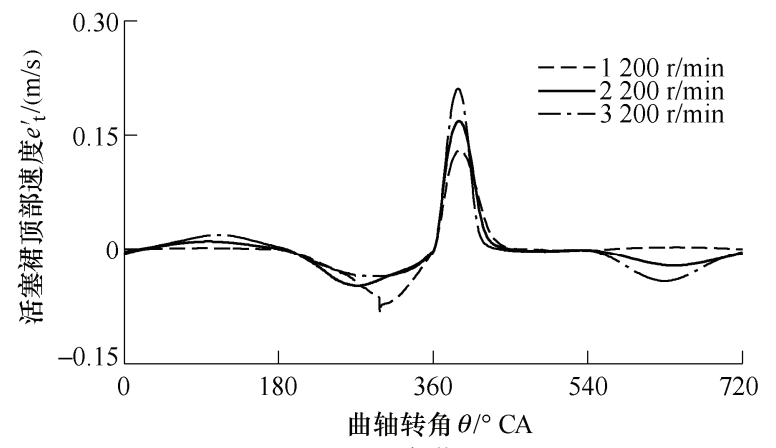

(a) 负荷 $0 \%$

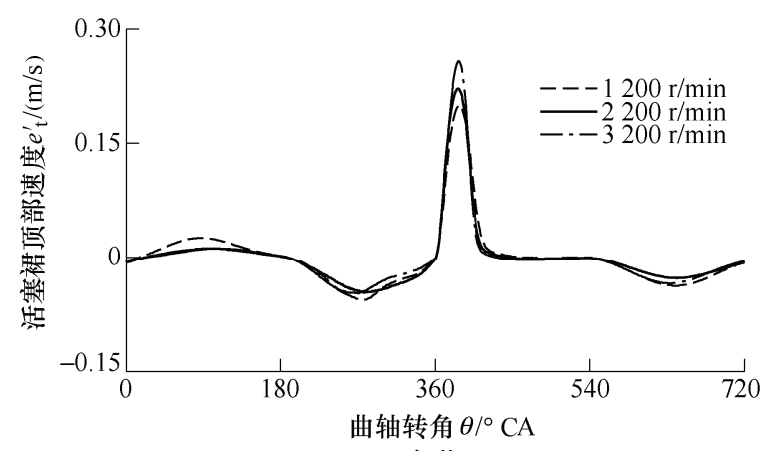

(b) 负荷 $60 \%$

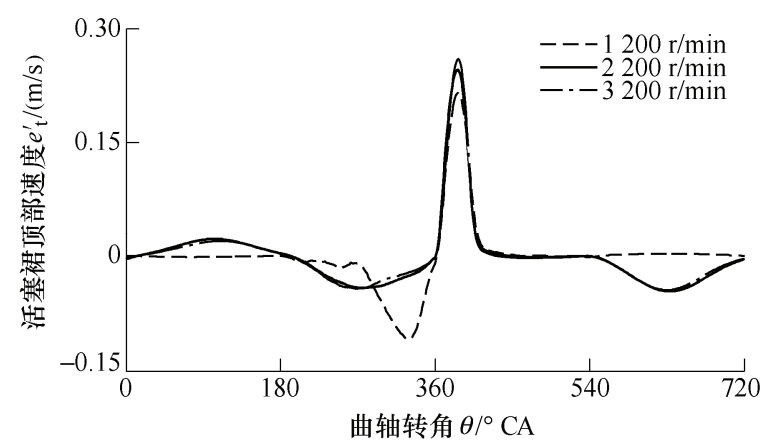

(c) 负荷 $100 \%$

图 5 活塞裙顶部速度

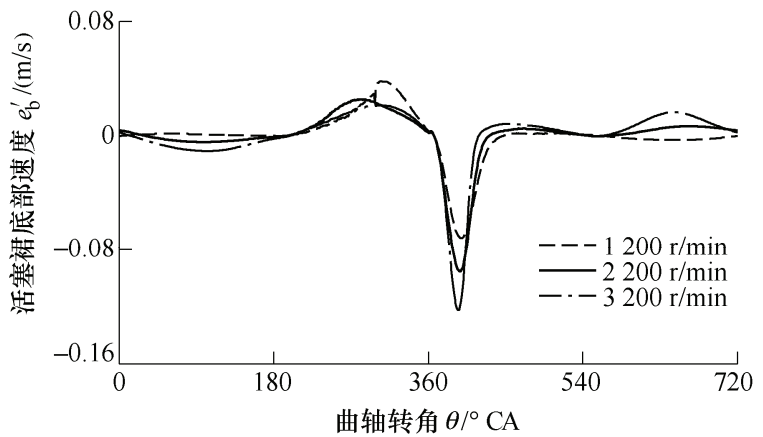

(a) 负荷 $0 \%$

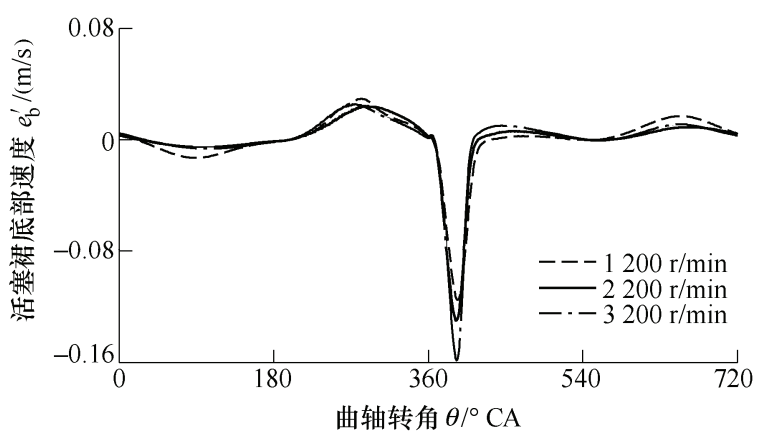

(b) 负荷 $60 \%$

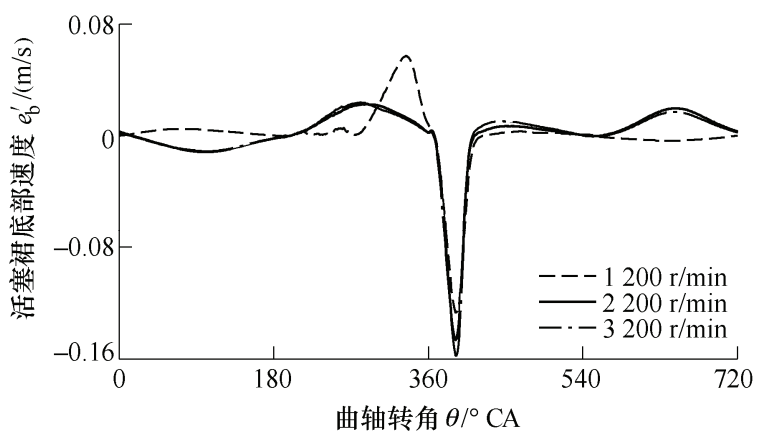

(c) 负荷 $100 \%$

图 6 活塞裙底部速度

如图 7 所示，在内燃机中等负荷(负荷 $60 \%$ ) 下，一个内燃机工作循环中活塞裙-缸套摩擦副最 小油膜厚度的变化趋势随转速的变化不明显; 在 内燃机小负荷(负荷 $0 \%$ ) 和全负荷(负荷 100\%)下， 中高转速 (2 $200 \mathrm{r} / \mathrm{min}$ 和 $3200 \mathrm{r} / \mathrm{min}$ ) 时最小油膜 厚度的变化趋势随转速的变化也不明显, 但是低 转速(1 $200 \mathrm{r} / \mathrm{min}$ )时一个内燃机工作循环中除燃 烧上止点附近外最小油膜厚度的变化趋势与中高 转速 (2 $200 \mathrm{r} / \mathrm{min}$ 和 $3200 \mathrm{r} / \mathrm{min}$ ) 时的差别却比较 明显。在内燃机中小负荷下，一个内燃机工作循 环中活塞裙-缸套摩擦副最小油膜厚度的最小值 一般都位于膨胀行程开始附近; 但是在内燃机全 负荷下, 低转速(1200 r $/ \mathrm{min})$ 时最小油膜厚度的最 小值出现在进气上止点附近, 中高转速 (2 200 $\mathrm{r} / \mathrm{min}$ 和 $3200 \mathrm{r} / \mathrm{min}$ ) 时最小油膜厚度的最小值出 现在膨胀行程结束附近。如表 2 所示, 在不同内 燃机负荷下，一个内燃机工作循环中活塞裙-缸套 摩擦副最小油膜厚度的最小值随转速变化没有一 
定的规律; 小负荷(负荷 $0 \%$ )下，中等转速(2 200 $\mathrm{r} / \mathrm{min}$ ) 时最小油膜厚度的最小值最小; 中等负荷 (负荷 $60 \%$ )下, 高转速(3 $200 \mathrm{r} / \mathrm{min}$ ) 时最小油膜厚 度的最小值最小; 全负荷下, 低转速(1 $200 \mathrm{r} / \mathrm{min})$ 时最小油膜厚度的最小值最小。

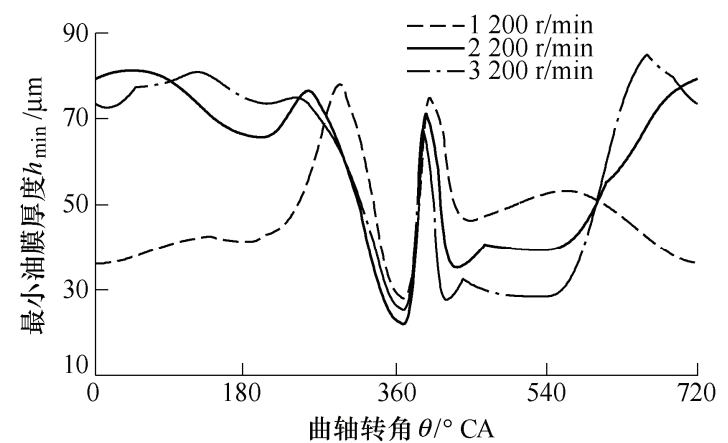

(a) 负荷 $0 \%$

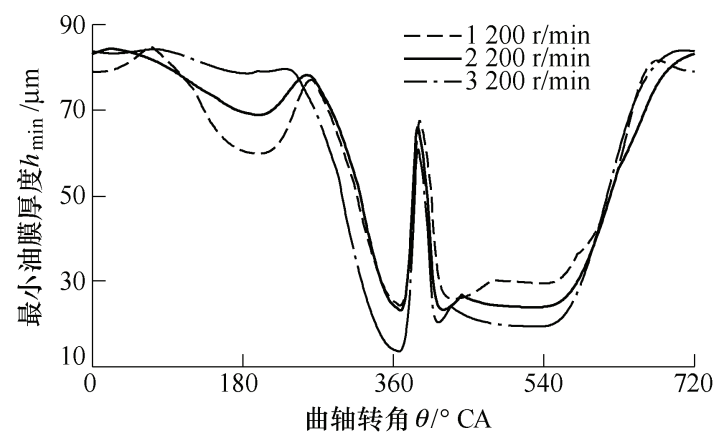

(b) 负荷 $60 \%$

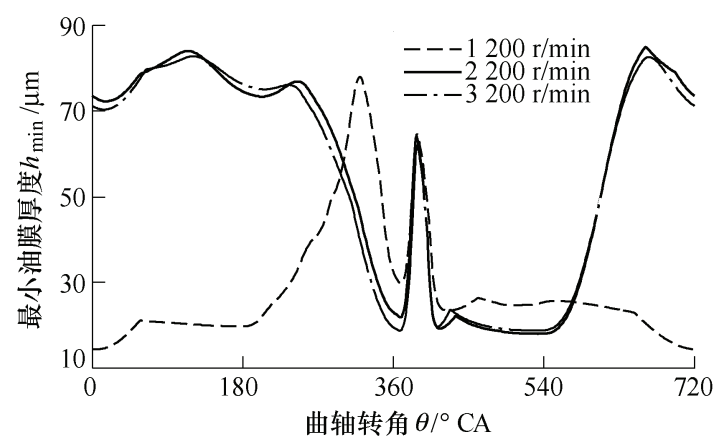

(c) 负荷 $100 \%$

图 7 最小油膜厚度

表 2 内燃机工作循环中最小油膜厚度的最小值 $\mu \mathrm{m}$

\begin{tabular}{cccc}
\hline \multirow{2}{*}{ 负荷 } & \multicolumn{3}{c}{ 最小油膜厚度 } \\
\cline { 2 - 4 } & $1200 \mathrm{r} / \mathrm{min}$ & $2200 \mathrm{r} / \mathrm{min}$ & $3200 \mathrm{r} / \mathrm{min}$ \\
\hline $0 \%$ & 27.98 & 21.97 & 25.27 \\
$60 \%$ & 24.24 & 23.11 & 13.49 \\
$100 \%$ & 14.33 & 17.98 & 18.68 \\
\hline
\end{tabular}

如图 8 所示, 在不同内燃机负荷下, 一个内燃 机工作循环中活塞裙-缸套摩擦副最大油膜压力随 转速的变化趋势基本一致, 且主要在膨胀行程中随 转速的变化数值差别较大, 其他时刻差别较小。在 内燃机中等负荷(负荷 60\%)和全负荷(负荷 100\%) 下, 一个内燃机工作循环中最大油膜压力的最大值 都位于膨胀行程前部; 在内燃机小负荷(负荷 $0 \%$ )
下, 中低转速 (1 $200 \mathrm{r} / \mathrm{min}$ 和 $2200 \mathrm{r} / \mathrm{min})$ 时最大 油膜压力的最大值出现在压缩行程后部, 高转速 (3 $200 \mathrm{r} / \mathrm{min}$ ) 时出现在膨胀行程前部。如表 3 所示, 在不同内燃机负荷下，一个内燃机工作循环中最大 油膜压力的最大值均随转速增加而增加。

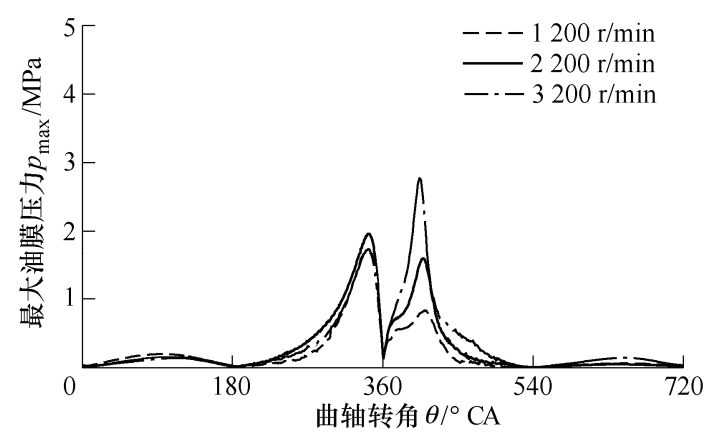

(a) 负荷 $0 \%$

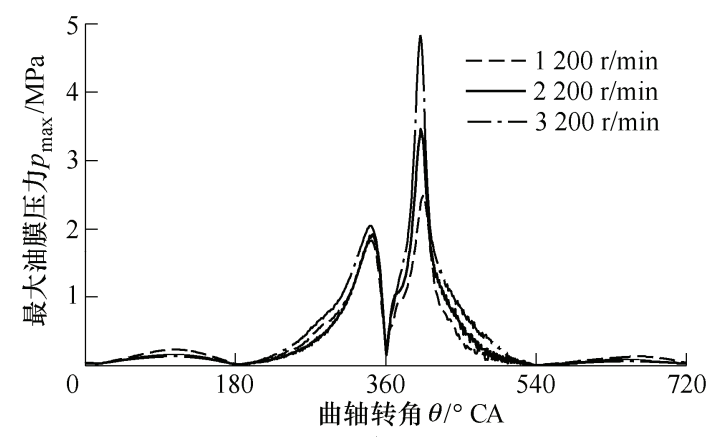

(b) 负荷 $60 \%$

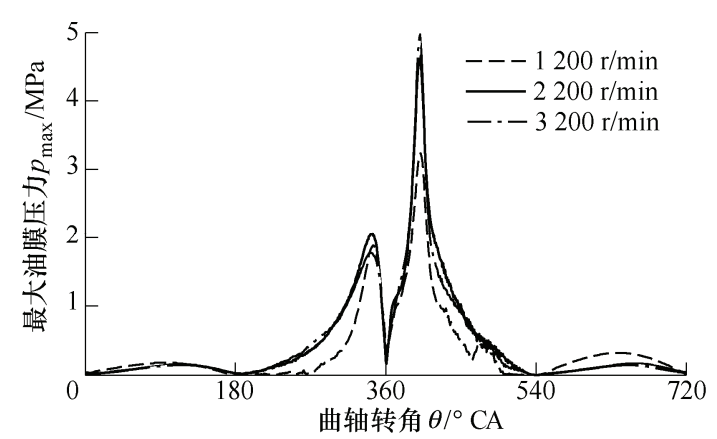

(c) 负荷 $100 \%$

图 8 最大油膜压力

表 3 内燃机工作循环中最大油膜压力的最大值 $\mathrm{MPa}$

\begin{tabular}{cccc}
\hline \multirow{2}{*}{ 负荷 } & \multicolumn{3}{c}{ 最大油膜压力 } \\
\cline { 2 - 4 } & $1200 \mathrm{r} / \mathrm{min}$ & $2200 \mathrm{r} / \mathrm{min}$ & $3200 \mathrm{r} / \mathrm{min}$ \\
\hline $0 \%$ & 1.72 & 1.96 & 2.77 \\
$60 \%$ & 2.50 & 3.46 & 4.82 \\
$100 \%$ & 3.24 & 4.65 & 4.97 \\
\hline
\end{tabular}

如图 9 所示，在不同内燃机负荷下，一个内燃 机工作循环中活塞裙-缸套摩擦副摩擦功率随转速 的变化趋势基本一致, 在各个行程中部摩擦功率随 转速的变化数值差别较大, 摩擦功率的最大值都位 于膨胀行程期间。如表 4 所示, 在不同内燃机负荷 下, 一个内燃机工作循环中摩擦功率平均值均随转 速增加而明显增加。 


\section{2 相同转速不同负荷时}

从图 3、4 可知, 内燃机中高转速 $(2200 \mathrm{r} / \mathrm{min}$ 和 $3200 \mathrm{r} / \mathrm{min}$ )下, 当负荷变化时, 工作循环不同时 刻活塞二阶运动横向位移的方向和大小基本一致, 活塞的摆动和倾斜方位基本相同; 只是在内燃机中 等转速(2 $200 \mathrm{r} / \mathrm{min}$ )较高负荷(负荷 100\%)和内燃机 高转速(3 $200 \mathrm{r} / \mathrm{min}$ )中等负荷(负荷 $60 \%$ )时, 进气行 程和排气行程中活塞横向位移的大小存在一定的不 同。但是, 内燃机低转速 $(1200 \mathrm{r} / \mathrm{min})$ 时, 不同负荷 下, 工作循环中不同时刻活塞横向位移存在较大不 同，将导致活塞的摆动和倾斜方位状况差别显著。

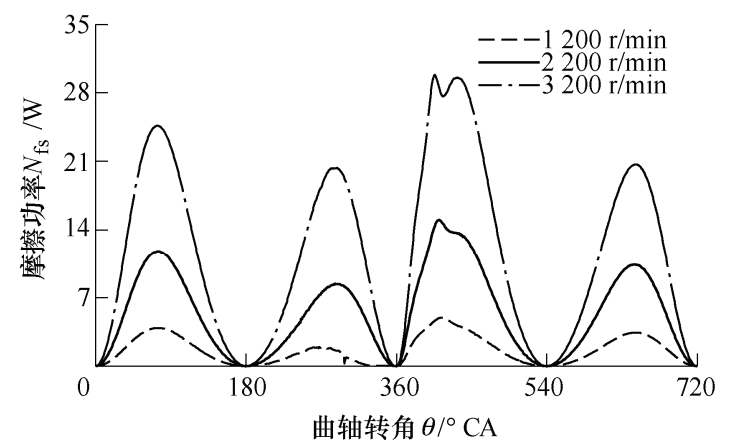

(a) 负荷 $0 \%$

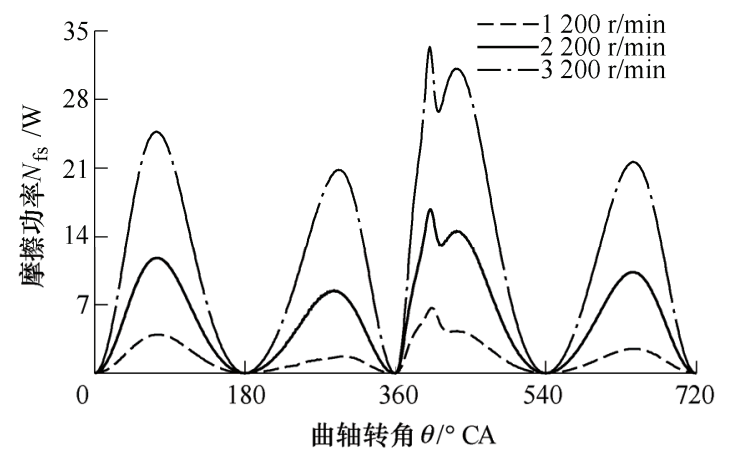

(b) 负荷 $60 \%$

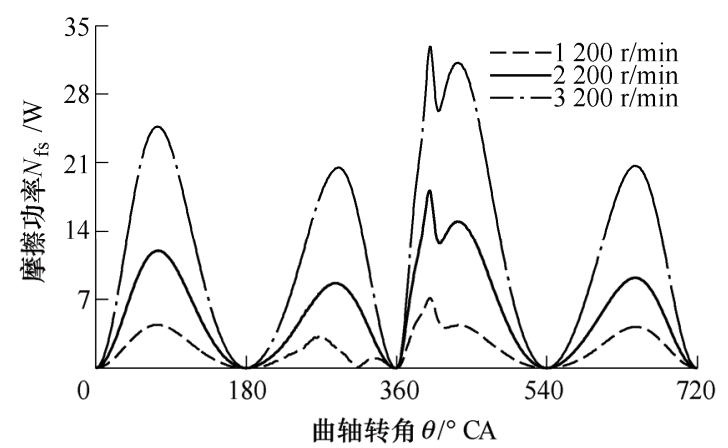

(c) 负荷 $100 \%$

图 9 摩擦功率

表 4 一个内燃机工作循环摩擦功率的平均值 W

\begin{tabular}{cccc}
\hline \multirow{2}{*}{ 负荷 } & \multicolumn{3}{c}{ 摩擦功率 } \\
\cline { 2 - 4 } & $1200 \mathrm{r} / \mathrm{min}$ & $2200 \mathrm{r} / \mathrm{min}$ & $3200 \mathrm{r} / \mathrm{min}$ \\
\hline $0 \%$ & 1.63 & 5.47 & 11.69 \\
$60 \%$ & 1.65 & 5.66 & 12.16 \\
$100 \%$ & 2.00 & 5.66 & 12.04 \\
\hline
\end{tabular}

由图 5、6 可见, 内燃机不同转速时, 不同负荷 下, 活塞二阶运动横向移动速度的变化趋势基本相 同。在不同内燃机转速下, 随着负荷增加, 活塞横 向移动速度的最大值都将增加, 将加剧活塞的摆动 和冲击。

如图 7 所示, 内燃机转速一定, 活塞裙-缸套摩 擦副最小油膜厚度随负荷的变化规律基本相同, 但 是在低转速时存在一定的差异。一个工作循环中最 小油膜的最小值一般位于膨胀行程开始附近，但是 内燃机低转速(1 $200 \mathrm{r} / \mathrm{min})$ 全负荷时出现在进气行 程开始附近, 中等转速 $(2200 \mathrm{r} / \mathrm{min}$ ) 全负荷时出现在 膨胀行程结束附近。如表 2 所示, 在不同内燃机转 速下，一个工作循环中最小油膜厚度的最小值随负 荷变化的规律不完全一致; 低转速(1 $200 \mathrm{r} / \mathrm{min}$ )时最 小油膜厚度的最小值随负荷增加而减小; 中低转速 (1 $200 \mathrm{r} / \mathrm{min}$ 和 $2200 \mathrm{r} / \mathrm{min}$ )下, 高负荷( $100 \%$ 负荷) 时最小油膜厚度的最小值最小; 高转速 (3 $200 \mathrm{r} / \mathrm{min}$ ) 下，中等负荷(负荷 $60 \%$ )时最小油膜厚度的最小值 最小。

从图 8 和表 3 可见, 内燃机转速相同时, 活塞 裙-缸套摩擦副最大油膜压力随负荷的变化规律基 本相同, 主要在膨胀行程中随转速的变化数值差别 较大, 其它时刻差别较小。除内燃机中低转速(1200 和 $2200 \mathrm{r} / \mathrm{min}$ ) 和小负荷(负荷 $0 \%$ )外，一个工作循环 中最大油膜压力的最大值一般都位于膨胀行程前 部。内燃机转速一定时, 随负荷增加最大油膜压力 的最大值增加。

由图 9 和表 4 可知, 内燃机转速相同时, 一个 工作循环中活塞裙-缸套摩擦副摩擦功率随负荷的 变化趋势基本一致, 各个行程中的摩擦功率和一个 工作循环中的平均摩擦功率随负荷的变化不明显。

\section{4 结论}

（1）虽然大部分内燃机工况下，活塞二阶运动 横向位移的最大值出现在燃烧上止点附近，但是在 内燃机负荷较高转速较低的工况下，活塞横向位移 的最大值位于进气上止点附近。

（2）内燃机负荷较小和较高时, 不同转速下, 进气 行程和排气行程中活塞二阶运动横向位移的方向和大 小存在明显不同。内燃机转速较低时, 不同负荷下, 工作循环中不同时刻活塞横向位移存在较大不同。

(3) 内燃机不同工况下, 活塞二阶运动横向移 动速度在一个内燃机工作循环中的变化趋势基本相 同，其最大值一般都出现在膨胀行程靠近燃烧上止 点附近。随着内燃机负荷和转速增加, 活塞横向移 
动速度的最大值增加。

(4) 内燃机不同工况下, 活塞裙-缸套摩擦副最 小油膜厚度在一个工作循环中的变化规律不尽相 同；一个工作循环中最小油膜厚度的最小值一般都 位于膨胀行程开始附近, 但是也存在不同情况。不 同内燃机负荷时, 一个工作循环中最小油膜厚度的 最小值随转速变化没有一定的规律; 不同内燃机转 速时, 一个工作循环中最小油膜厚度的最小值随负 荷变化的规律也不完全一致。一个工作循环中最小 油膜厚度的最小值出现在高转速中等负荷和低转速 全负荷情况下。

(5) 在不同工况下, 一个内燃机工作循环中活 塞裙-缸套摩擦副最大油膜压力随负荷的变化规律 基本相同; 最大的油膜压力一般都出现在膨胀行程 的前期。内燃机负荷一定时, 最大的油膜压力随转 速增加而增加; 内燃机转速一定时, 随负荷增加最 大的油膜压力增加。

（6）内燃机负荷相同时, 各个行程中的活塞裙缸套摩擦副摩擦功率和一个内燃机工作循环中的平 均摩擦功率随转速增加均增加明显; 内燃机转速相 同时, 各个行程中的摩擦功率和一个工作循环中的 平均摩擦功率随负荷变化不明显。

综上所述，不同内燃机工况下的活塞二阶运动 和活塞裙-缸套摩擦副润滑性能存在差异, 最不利情 况不一定出现在内燃机标定工况。这表明, 内燃机 活塞组件设计时, 应考虑内燃机的实际运动和受力, 分析不同工况下的活塞二阶运动和活塞裙-缸套摩 擦副润滑性能。

\section{参 考 文 献}

[1] DURSUNKAYA Z, KERIBAR R, GANAPATHY V. A model of piston secondary motion and elastohydrodynamic skirt lubrication[J]. ASME Journal of Tribology, 116(4), 1994: 777-785.

[2] SHAH P, MOURELATOS Z P, PATEL P. Piston secondary dynamics considering elastohydrodynamic lubrication[C]// SAE World Congress, April 10-12, 2007, Cobo Center, Detroit, Michigan, USA. SAE : 2007-01-1251.

[3] 杨俊伟, 于旭东, 王成奉, 等. 考虑活塞热变形的活塞 裙部润滑计算分析 [J]. 内燃机学报, 2002, 20(4): 365-368.

YANG Junwei, YU Xudong, WANG Chengtao, et al. Calculation and analysis of piston skirt lubrication considering the thermal deformation of piston[J]. Transactions of CSICE, 2002, 20(4): 365-368.

[4] 阮登芳, 高金金, 马克. 缸套弹性变形对活塞二阶运动和
裙部润滑特性的影响 $[\mathrm{J}]$. 汽车工程, 2015, 37(5): 571-575.

RUAN Dengfang, GAO Xin, MA Ke. Effects of liner elastic deformation on piston 2nd-order motion and piston skirt lubrication characteristics[J]. Automotive Engineering, 2015, 37(5): 571-575.

[5] MANSOURI S H, WONG V W. Effects of piston design parameters on piston secondary motion and skirt-liner friction[J]. Proc IMechE Part J: Journal of Engineering Tribology, 2005, 219(6): 435-449.

[6] GUNELSU O, AKALIN O. The Effects of piston skirt profiles on secondary motion and friction[J]. ASME Journal of Engineering for Gas Turbines and Power, 2014, 136(6): 062503-1-8.

[7] 王政, 唐建, 于旭东, 等. 活塞裙部型线对活塞系统二 阶运动和摩擦功率的影响 [J]. 内燃机学报, 1999, 17(4): 383-387.

WANG Zheng, TANG Jian, YU Xudong, et al. The influence of piston skirt profile on the secondary motion and friction power loss of piston[J]. Transactions of CSICE, 1999, 17(4): 383-387.

[8] LIU K, XIE Y B, GUI C L. A comprehensive study of the friction and dynamic motion of the piston assembly[J]. Proc IMechE Part J: Journal of Engineering Tribology, 1998, 212(3): 221-226.

[9] MALAGI R R, KURBET S N, KRISHNAKUMAR R. A comprehensive model to study the dynamic motion of piston and friction and lubrication in IC engine $[\mathrm{C}] / / \mathrm{SAE}$ World Congress, April 10-12, 2008, Cobo Center, Detroit, Michigan, USA. SAE: 2008-28-0061.

[10] ZHANG Z, XIE Y, ZHANG X, et al. Analysis of piston secondary motion considering the variation in the system inertia[J]. Proc IMechE Part D: Journal of Automobile Engineering, 2009, 223(4): 549-563.

[11] 张执南, 张效翔, 李响, 等. 活塞二阶运动及活塞裙部 摩擦的瞬态分析[J]. 摩擦学学报, 2010, 30(2): 184-189. ZHANG Zhinan, ZHANG Xiaoxiang, LI Xiang, et al. Transient analysis of piston secondary motion and piston skirt friction[J]. Tribology, 2010, 30(2): 184-189.

[12] MURAKAMI H, NAKANISHI N, ONO N, et al. New three-dimensional piston secondary motion analysis method coupling structure analysis and multi body dynamics analysis[J]. SAE International Journal of Engines, 2011, 5(1): 42-50.

[13] MENG X, FANG C, XIE Y. Transient tribodynamic model of piston skirt-liner systems with variable speed effects[J]. Tribology International, 2016, 94: 640-651.

[14] ZHAO B, DAI X D, ZHANG Z N, et al. A new numerical method for piston dynamics and lubrication analysis[J]. 
Tribology International, 2016，94: 395-408.

[15] KNOLL G D, PEEKEN H J. Hydrodynamic lubrication of piston skirts $[\mathrm{J}]$. ASME Journal of Lubrication Technology, 1982, 104(4): 504-508.

[16] LI D F, RHODE S M, EZZAT H A. An automotive piston lubrication model[J]. ASLE Transactions, 1983, 26(2): 151-160.

[17] ZHU D, CHENG H S, ARAI T, et al. A numerical analysis for piston skirts in mixed lubrication - Part I: basic modeling[J]. ASME Journal of Tribology, 1992, 114: 553-562.

[18] ZHU D, CHENG H S, ARAI T, et al. A numerical analysis for piston skirts in mixed lubrication - Part II: deformation considerations[J]. ASME Journal of Tribology, 1993, 115: 125-133.

[19] NING L, MENG X, XIE Y. Incorporation of deformation in a lubrication analysis for automotive piston skirt-liner system[J]. Journal of Engineering Tribology, 2013, 227(6): 654-670.

[20] LITTLEFAIR B, CRUZ M D L, MILLS R, et al. Lubrication of a flexible piston skirt conjunction subjected to thermo-elastic deformation - A combined numerical and experimental investigation[J]. Journal of Engineering Tribology, 2014, 228(1): 69-81.

[21] 武涁, 宁李谱, 孟祥慧, 等. 汽车发动机活塞裙部-缸 套系统摩擦学仿真分析和减摩设计 [J]. 摩擦学学报, 2012, 32(6): 577-583.

WU Bin, NING Lipu, MENG Xianghui, et al. Tribological simulation and design of piston skirt-liner system to reduce friction of automotive engines[J]. Tribology, 2012, 32(6): 577-583.

[22] 余志壮, 宋正华, 董光能, 等. 内燃机气缸套失圆对活 塞动压润滑和摩擦特性的影响 [J]. 摩擦学学报, 2005, 25(3): 243-247.

YU Zhizhuang, SONG Zhenghua, DONG Guangneng, et al. Influence of cylinder liner out-of-roundness on dynamic lubrication and friction characteristics of piston[J]. Tribology, 2005, 25(3): 243-247.

[23] MENG F M, ZHANG Y Y, HU Y Z, et al. Thermo-elasto-hydrodynamic lubrication analysis of piston skirt considering oil film inertia effect[J]. Tribology International, 2007, 40: 1089-1099.

[24] QASIM S A, CHAUDHRI U F, MALIK M A. Analyzing viscoelastic effects in piston skirts EHL at small radial clearances in initial engine start up[J]. Tribology International, 2012, 45: 16-29.

[25] QASIM S A, MALIK M A, KHAN M A, et al. Modeling shear heating in piston skirts EHL considering different viscosity oils in initial engine start up[J]. ASME Journal of Engineering for Gas Turbines and Power, 2012, 134: 032802-1-8.

[26] MAHDAVI S H, MANSOURI S H, KIMIAEIFAR A. Effects of using smart fluid in lubrication on skirt-liner friction[J]. Industrial Lubrication and Tribology, 2012, 64(2): 90-97.

[27] NING L, MENG X, XIE Y. Effects of lubricant shear thinning on the mixed lubrication of piston skirt-liner system[J]. Journal of Mechanical Engineering Science, 2013, 227(7): 1585-1598.

[28] MENG X, NING L, XIE Y, et al. Effects of the connecting-rod-related design parameters on the piston dynamics and the skirt-liner lubrication[J]. Journal of Automobile Engineering, 2013, 227: 885-898.

[29] PATIR N, CHENG H S. An average flow model for determining effect of three dimensional roughnesses on partial hydrodynamic lubrication[J]. ASME Journal of Lubrication Technology, 1978, 100(1): 12-17.

[30] PATIRr N, CHENG H S. Application of average flow model to lubrication between rough sliding surfaces[J]. ASME Journal of Lubrication Technology, 1979, 101(2): 220-230.

[31] GREENWOOD J A, TRIPP J H. The contact of two nominally flat rough surfaces[J]. Proc. Instn. Mech. Engrs., 1970, 185: 625-633.

[32] 许贤良, 王开松, 孟利民. 流体力学[M]. 2 版. 北京: 国防工业出版社, 2011.

XU Xianliang, WANG Kaisong, MENG Limin. Fluid dynamics[M]. 2nd ed. Beijing: National Defence Industry Press, 2011.

[33] 李庆扬, 莫孜中, 祁力裙. 非线性方程的数值解法 [M]. 北京: 科学出版社, 1999.

LI Qingyang, MO Zizhong, QI Liqun. Numerical method of nonlinear equation[M]. Beijing: Science Press, 1999.

作者简介: 孙军, 男, 1960 年出生, 博士, 教授, 博士研究生导师。主 要研究方向为摩擦学和现代设计理论与方法, 发表论文 150 余篇。 E-mail: sunjun_hfut@163.com 